\title{
Simultaneous bilateral subcutaneous pyelovesical bypass as a salvage procedure in refractory retroperitoneal fibrosis
}

\author{
Nicholas Haddad, MSc; Sero Andonian, MD, MSc, FRCSC; Maurice Anidjar, MD, PhD
}

Division of Urology, Department of Surgery, McGill University Health Centre, McGill University, Montreal, QC

Cite as: Can Urol Assoc J 2013;7(5-6):e417-20. http://dx.doi.org/10.5489/cuaj.1395 Published online June 12, 2013.

\section{Abstract}

Ureteral obstruction causing renal failure is the most common complication associated with retroperitoneal fibrosis (RPF). Initial management includes steroid therapy together with ureteral stenting. When these fail, ureterolysis is the recommended surgical procedure. However, this could be challenging and recurrence is common. The aim of the present case series was to assess the feasibility of inserting simultaneous bilateral subcutaneous pyelovesical bypass grafts (SPBGs) in patients presenting with RPF who had failed initial endourologic/surgical management.

\section{Introduction}

Retroperitoneal fibrosis (RPF) is a rare disease characterized by chronic inflammation and fibrosis in the retroperitoneum. The idiopathic form of this disorder accounts for $70 \%$ percent of cases; the remaining $30 \%$ is secondary to a variety of causes, including neoplasms, radiation therapy, surgery, infection and certain medications. ${ }^{1,2}$ In a Dutch study, the annual incidence of idiopathic disease was 1.3 per 100 000, with a 3.3:1.0 male-female ratio. ${ }^{3}$ The fibro-inflammatory tissue characteristic of this disease usually surrounds the abdominal aorta and the iliac arteries, extending into the retroperitoneum to encase neighboring structures. ${ }^{2}$ Ureteral obstruction with secondary acute or chronic renal failure is the most common and severe complication associated with RPF, with ureteral involvement reported in $80 \%$ to $100 \%$ of cases. ${ }^{4,5}$

Generally, the goals of therapy are to relieve obstruction and to slow or prevent the progression of fibrosis. Initial treatment of urinary tract obstruction involves steroid therapy together with the placement of indwelling double pigtail stents or percutaneous nephrostomy tubes. ${ }^{2}$ In refractory cases, ureterolysis followed by omental wrapping can be performed either through open, laparascopic or robotic surgery, with renal autotransplantation traditionally left as a last resort option. ${ }^{6-9}$ Surgical intervention carries the risk of intraoperative morbidity (i.e., injuries to the ureter, renal pelvis and iliac vein); complications, such as urinary tract infection, prolonged ileus and renal insufficiency, have also been reported. ${ }^{9,10}$ Ureteral obstruction recurs in about $10 \%$ of patients who are concomitantly treated with steroids, but can rise up to $50 \%$ in those who undergo surgery alone. ${ }^{11}$ Therefore, a definitive treatment for some patients may remain elusive even after surgical intervention.

Urinary diversion through subcutaneous pyelovesical bypass grafts (SPBG) was initially used in the palliative treatment of pelvic malignancies as an alternative to permanent percutaneous nephrostomy. ${ }^{12}$ In cases of irreversible ureteral obstruction following renal transplantation, SPBG has been shown to be a safe and efficient alternative to open surgery. ${ }^{13,14}$ The SPBG is composed of a silicone tube enveloped by high-porosity polyester (Detour Coloplast, Denmark). This approach is successful in salvaging renal grafts when endourologic or open management fails, resulting in stable renal function and no evidence of obstruction. ${ }^{14,15}$ After a mean follow-up of 6 years (range: 1-14) in 7 patients who had received an SPBG, no obstruction, encrustation or kinking was observed; only 1 patient required removal of the prosthesis due to Candidal infection. ${ }^{16}$ We present 3 cases of RPF with bilateral ureteral obstruction that were treated with simultaneous bilateral SPBGs as a last resort.

\section{Operative procedure}

Under general anesthesia, a 3-way Foley catheter was inserted and approximately 300 cc of saline fluid was injected into the bladder. Patients were then placed in a lateral decubitus position at 45 degrees with either the left or right side up. After prepping and draping the flank and suprapubic areas, a nephrostogram was performed to ensure access to the lower calyx, through already established nephrostomy tracts. Guidewires were then placed and the tract was dilated to 
30-French with an X-FORCE U30 balloon (Bard Medical Division, Covington, GA) and a 30-French Amplatz sheath was subsequently placed inside the collecting system. Next, a lateralized Pfannenstiel incision 5-cm in length was made 2 fingerbreadths above the symphysis pubis, and this incision was then deepened through the subcutaneous tissues. The rectus abdominis muscle was separated and the retroperitoneal space developed such that the bladder was reached and dissected off the detrusor muscle. Two stay sutures were made transmurally to identify the future site of anastamosis. A subcutaneous tract was then created from the initial nephrostomy tube incision to the bladder site.

The SPBG was then inserted through the Amplatz sheath under fluoroscopy such that a radiopaque mark sits between the calyx and renal parenchyma (Fig. 1). The Amplatz sheath was then removed. The SPBG was passed inside the tunneler until it was re-exposed through the abdmoninal incision. The distal end of the SPBG was then tapered distally such that a $3-\mathrm{cm}$ stretch of silicone alone would be found within the bladder. Four 3-0 Vicryl sutures were then placed transmurally within the bladder and through the distal end of the polyester sheath and then tightened. Abdominal fascia was then closed with PDS 1 sutures and the skin closed with staples. Kidney incisions were closed with Vicryl and with skin clips. The same procedure was then performed on the contralateral side. The Foley catheter was removed 10 days postoperatively after a cystogram confirming bladder healing.

\section{Case 1: Results}

A 52-year-old female presented with idiopathic RPF encasing the abdominal aorta, inferior vena cava and proximal ureters. This resulted in bilateral ureteral obstruction and hydronephrosis generating a creatinine of $111 \mathrm{umol} / \mathrm{L}$ and an estimated glomerular filtration rate (eGFR) at $48 \mathrm{~mL} /$ $\mathrm{min} / 1.73 \mathrm{~m}^{2}$ (all eGFR values calculated using the extended modification of diet in renal disease equation). The obstruction was initially treated with double pigtail stents, but this failed to relieve symptoms and nephrostomy tubes were subsequently inserted. Bilateral ureterolysis was performed, but ureters could not be wrapped in omentum or intraperitonealized. Surgery eventually failed and shortly thereafter the patient developed severe urosepsis with fever and associated generalized tonic-clonic seizures. A decision was then made to attempt bilateral SPBG insertion. Immediately after the procedure, a urine leak was discovered at the site of kidney incision on the right side. This was found to be due to compression of the silicon portion of the graft at its insertion site in the bladder. The patient was brought back to the operating room within 24 hours and the SPBG was re-anastamosed to the bladder using UR-6 needles to ensure its proper placement. A distal urine leak subsequently appeared at the bladder site prompting the insertion of nephrostomy tubes to reduce the volume of urine reaching the bladder. The leakage ceased within 24 hours, and 7 days later the nephrostomy tubes were removed following a nephrostogram that showed no extravasation. At 15-months of follow-up, renal function has stabilized with creatinine sitting at $86 \mathrm{umol} / \mathrm{L}$ and eGFR at $64 \mathrm{~mL} / \mathrm{min} / 1.73 \mathrm{~m}^{2}$ (Table 1 ), and computed tomography scan showed no hydronephrosis (Fig. 1). The patient has not had a urinary tract infection (UTI) since the operation, and claims to have a significantly improved quality of life.

\section{Case 2: Results}

A 44-year-old female presented with severe bilateral hydronephrosis and progressive renal failure, with a creatinine of $285 \mathrm{umol} / \mathrm{L}$ and an eGFR of $17 \mathrm{~mL} / \mathrm{min} / 1.73 \mathrm{~m}^{2}$. The patient was diagnosed with colorectal cancer, for which she received radiotherapy as part of her treatment. Exposure to radiation led to the development of RPF that encased both ureters causing obstruction. Initially she was treated with bilateral ureteral catheters. This regimen was not able to definitively alleviate the obstruction, with the patient experiencing two episodes of acute renal failure (creatinine $>1200$ umol/L), spiking fevers and urosepsis. Following blood cultures positive for Streptococcus, nephrostomy tubes were inserted while a definitive treatment for her obstruction was sought. Given the option of ureterolysis or SPBG insertion, the patient chose the latter. Bilateral SPBG insertion was performed with no immediate postoperative complications.

Seven months after graft insertion, she developed urolithiasis in the prosthesis on the right side causing acute renal failure with a creatinine of over $700 \mathrm{umol} / \mathrm{L}$. The stone was removed via flexible ureteroscopy and basket extraction through the SPBG, and the serum creatinine returned to baseline levels. The patient also developed 2 episodes of urosepsis caused by Candida colonization at

\begin{tabular}{|c|c|c|c|c|c|c|c|}
\hline Case & Age & Etiology & $\begin{array}{l}\text { Follow-up } \\
\text { (months) }\end{array}$ & $\begin{array}{l}\text { Pre-SPBG Cr } \\
\text { (umol/L) }\end{array}$ & $\begin{array}{l}\text { Post-SPBG Cr } \\
\text { (umol/L) }\end{array}$ & $\begin{array}{l}\text { Pre-SPBG eGFR* } \\
\left(\mathrm{ml} / \mathrm{min} / 1.73 \mathrm{~m}^{2}\right)\end{array}$ & $\begin{array}{l}\text { Post-SPBG eGFR* } \\
\left(\mathrm{ml} / \mathrm{min} / 1.73 \mathrm{~m}^{2}\right)\end{array}$ \\
\hline 1 & 52 & Idiopathic & 15 & 111 & 86 & 48 & 64 \\
\hline 2 & 44 & Radiotherapy & 22 & 285 & 300 & 17 & 16 \\
\hline 3 & 78 & Radiotherapy & 18 & 138 & 159 & 34 & 29 \\
\hline
\end{tabular}

All eGFR values calculated using the extended MDRD equation. SPBG: Subcutaneous pyelovesical bypass graft; Cr: creatinine; eGFR: estimated glomerular filtration rate. 


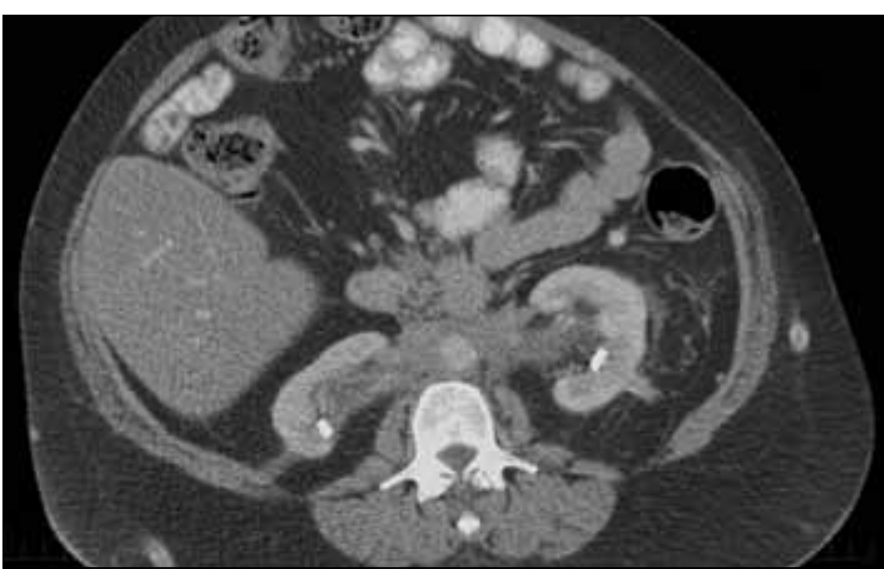

Fig. 1. A computed tomography scan taken at the 14-month follow-up revealed bilateral resolution of hydronephrosis in the presence of a persistent retroperitoneal mass encasing the aorta and inferior vena cava. The subcutaneous pyelovesical bypass grafts remain properly positioned as the radiopaque mark of the prosthesis can be found between the renal parenchyma and calyx bilaterally.

12 and 18 months postoperatively. Bilateral ureteroscopy was performed to remove obstructive fungal balls and she was treated for Candidal infection. At 13 months postopearatively, the patient presented with a UTI and a subcutaneous abscess on the right flank that grew Group B Streptococcus, Streptococcus anginosus and Bacteroides species. The abscess was percutaneously drained and treated. No leaks from either graft were found, and no other complications were reported. At the 22-month follow-up, her baseline creatinine stabilized at $300 \mathrm{umol} / \mathrm{L}$ and eGFR $16 \mathrm{~mL} / \mathrm{min} / 1.73 \mathrm{~m}^{2}$. The patient is currently undergoing elective ureteroscopy at 4-month intervals to detect early signs of obstruction and remove fungus balls when present.

\section{Case 3: Results}

A 78-year-old female with a history of colorectal cancer treated with a total colectomy and radiotherapy presented with bilateral hydronephrosis secondary to ureteral obstruction caused by RPF. Creatinine was at $138 \mathrm{umol} / \mathrm{L}$ and eGFR at $34 \mathrm{~mL} / \mathrm{min} / 1.73 \mathrm{~m}^{2}$. Double pigtail stents were inserted, but hydronephrosis persisted and renal function continued to deteriorate. She was deemed not suitable for ureterolysis, and nephrostomy tubes were inserted in preparation for SPBG graft placement (Fig. 2). There were no immediate postoperative complications, but the patient did develop 2 episodes of left-sided pyelonephritis. Candida was detected in urine cultures 14 and 18 months after the SPBGs were inserted, for which she was treated. After 18 months of follow-up, creatinine sits at $159 \mathrm{umol} / \mathrm{L}$ with eGFR at $29 \mathrm{~mL} / \mathrm{min} / 1.73 \mathrm{~m}^{2}$. Mild hydronephrosis persisted bilaterally, but her kidney function was stable. The patient is currently receiving Apo-Sulfatrim to prevent future episodes of infection.

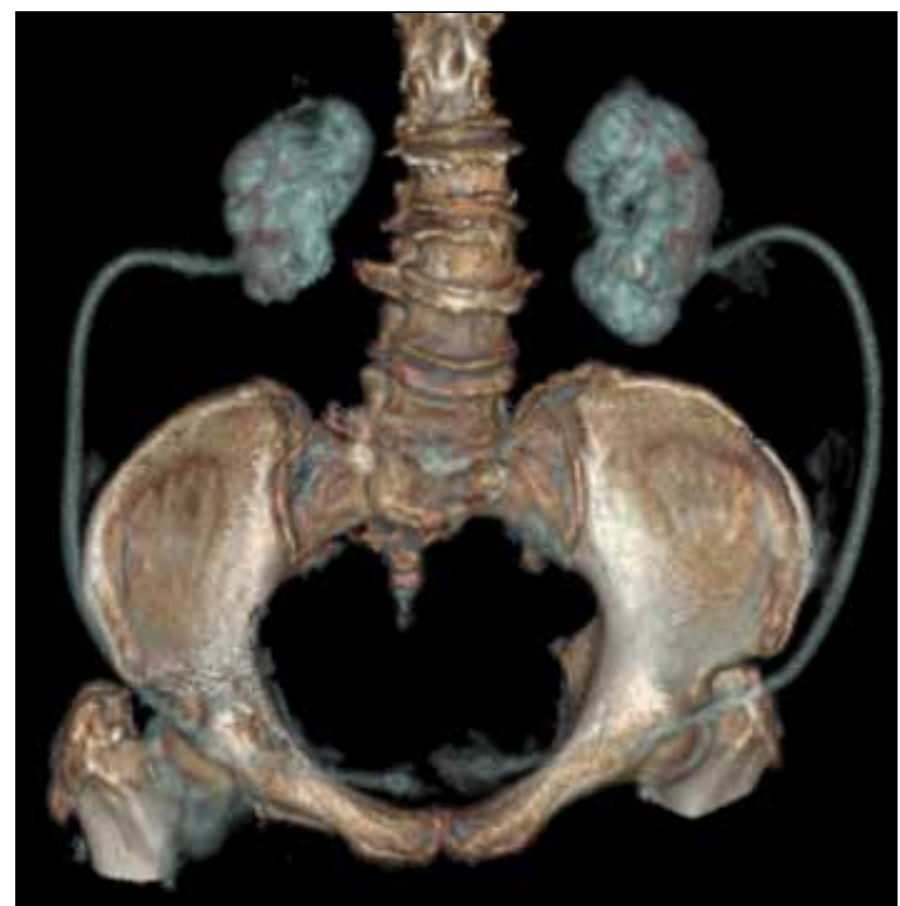

Fig. 2. A 3D-reconstruction using computed tomography-derived images shows bilateral subcutaneous pyelovesical bypass graft implants.

\section{Discussion}

We describe for the first time the bilateral insertion of SPBG as a treatment for ureteral obstruction in RPF. At a mean follow-up of 18.3 months, all 6 units remain functional and renal function stabilized with significant patient satisfaction. However, this procedure is not without limitations, which include misplacement of the prosthesis, infections and stones.

In Case 1, distal compression of the prosthesis was discovered during the immediate postoperative period. Re-insertion was performed the following day without long-term consequences. In a previous study involving renal transplant recipients, 2 out of 8 patients developed distal dislodgement following SPBG insertion with successful reinsertion. ${ }^{15}$ As all 3 patients in the present study received bilateral grafts simultaneously, the risks of such complications may have been elevated. Staging SPBG insertions could thus prove beneficial in reducing the risk of similar complications.

Recurrent infections are another limitation of the SPBGs. After a mean follow-up of 18.3 months, 1 patient developed 2 episodes of pyelonephritis requiring long-term prophylactic antibiotics. Two patients also developed Candidal colonization, with Case 2 requiring ureteroscopic intervention to relieve obstruction caused by a fungal ball. In a previous study involving kidney transplant recipients, an infection rate of $25 \%(2 / 8)$ was seen after a mean followup of 19.4 months, ${ }^{15}$ while another group in France found 
a rate of $42 \%(3 / 7)$ after a mean follow-up of 6 years. ${ }^{16}$ In the latter group, bacterial colonization of the SPBG was asymptomatic, while fungal colonization led to 1 death following removal of the prosthesis in this immunosuppressed patient. In our series, all 6 grafts inserted remain operational and well-tolerated by patients, who now claim a significant improvement in their quality of life. However, fungal colonization may be difficult to fully eradicate and prophylactic treatment is sometimes required.

The third limitation of SPBGs is the formation of stones, which occurred in 1 out of the 6 implanted renal units. Fortunately, SPBG implants are easily accessible to flexible ureteroscopy, thus allowing for the removal of potential obstructive stones or fungus balls. Interestingly, other studies involving renal transplant recipients with follow-up of to 15 years had no cases of encrustation or obstruction. ${ }^{15-17}$ Perhaps the encrustations/stones seen in Case 2 may have been related to chronic fungal infections. Despite these infections, renal function remained stable in this patient.

\section{Conclusion}

We present 3 cases of simultaneous bilateral SPBG insertion as a treatment for urinary obstruction in refractory cases of RPF. After a mean follow-up of 18.3 months, all 3 patients had stable renal function and a resolution of bilateral hydronephrosis, in addition to a significantly improved quality of life. Nevertheless, long-term follow-up is required before establishing this procedure as a valid alternative to conventional surgical management.

Competing interests: None declared.

This paper has been peer-reviewed.

\section{References}

1. Koep L, Zuidema GD. The clinical significance of retroperitoneal fibrosis. Surgery 1977;81:250-7.

2. Vaglio A, Salvarani C, Buzio C. Retroperitoneal fibrosis. Lancet 2006;367:241-51. http://dx.doi.org/10.1016/S0140-6736(06)68035-5

3. van Bommel EF, Jansen I, Hendriksz TR, et al. Idiopathic retroperitoneal fibrosis: prospective evaluation of incidence and clinicoradiologic presentation. Medicine (Baltimore) 2009;88:193-201. http://dx.doi.org/10.1097/MD.0b013e3181afc420

4. Baker LR, Mallinson WJ, Gregory MC, et al. Idiopathic retroperitoneal fibrosis. A retrospective analysis of 60 cases. Br J Urol 1987;60:497-503. http://dx.doi.org/10.1111/i.1464-410X.1987.tb05028.x

5. Vaglio A, Corradi D, Manenti L, et al. Evidence of autoimmunity in chronic periaortitis: a prospective study. Am J Med 2003;1 14:454-62. http://dx.doi.org/10.1016/S0002-9343(03)00056-1

6. Oesterwitz H, Lenk S, Hengst E, et al. Renal autotransplantation for idiopathic retroperitoneal fibrosis. Int Urol Nephrol 1994;26:167-71. http://dx.doi.org/10.1007/BF02768281

7. Lodde M, Pycha A, Palermo $S$, et al. Uretero-ureterocutaneostomy (wrapped by omentum). BJU Int 2005;95:371-3. http://dx.doi.org/10.1111/i.1464-410X.2005.05302.x

8. Simone G, Leonardo C, Papalia R, et al. Laparoscopic ureterolysis and omental wrapping. Urology 2008;72:853-8. http://dx.doi.org/10.1016/i.urology.2008.06.011

9. Seixas-Mikelus SA, Marshall SJ, Stephens DD, et al. Robot-assisted laparoscopic ureterolysis: case report and literature review of the minimally invasive surgical approach. JSLS 2010;14:313-9. http://dx.doi.org/10.4293/108680810X12785289145088

10. Srinivasan AK, Richstone L, Permpongkosol S, et al. Comparison of laparoscopic with open approach for ureterolysis in patients with retroperitoneal fibrosis. J Urol 2008;179:1875-8. http://dx.doi.org/10.1016/i.juro.2008.01.030

11. Marcolongo R, Tavolini IM, Laveder F, et al. Immunosuppressive therapy for idiopathic retroperitoneal fibrosis: a retrospective analysis of 26 cases. Am J Med 2004;116:194-7. htrtp://dx.doi.org/10.1016/.amimed.2003.08.033

12. Desgrandchamps $F$, Cussenot 0 , Meria $P$, et al. Subcutaneous urinary diversions for palliative treatment of pelvic malignancies. J Urol 1995;154(2 Pt 1):367-70.

13. Desgrandchamps $F$, Duboust $A$, Teillac $P$, et al. Total ureteral replacement by subcutaneous pyelovesical bypass in ureteral necrosis after renal transplantation. Transpl Int 1998;11:S150-1.

14. Andonian S, Zorn KC, Paraskevas $S$, et al. Aritificial ureters in renal transplantation. Urology 2005;66:1109. http://dx.doi.org/10.1016/i.urology.2005.05.012

15. Azhar RA, Hassanain $M$, Alifffry $M$, et al. Successful salvage of kidney allograffts threatened by ureteral stricture using pyelovesical bypass. Am J Transplant 2010;10:1414-9. http://dx.doi.org/10.1111/1.1600-6143.2010.03137.x

16. Muller CO, Meria P, Desgrandchamps F. Long-term outcome of subcutaneous pyelovesical bypass in extended ureteral stricture after renal transplantation. J Endourol 2011;25:1389-92. http://dx.doi.org/10.1089/end.2011.0085

17. Jabbour ME, Desgrandchamps F, Angelescu E, et al. Percutaneous implantation of subcutaneous prosthetic ureters: long-term outcome. J Endourol 2001;15:61 1-4. http://dx.doi.org/10.1089/089277901750426391

Correspondence: Dr. Maurice Anidjar, Assistant Professor, McGill University Health Centre, 687 Pine Ave West, Montreal, QC H3A 1A1; fax: 514-843-1552; maurice.anidjar@mcgill.ca 